\title{
Intratumoral heterogeneity of glutaminase and lactate dehydrogenase A protein expression in colorectal cancer
}

\author{
YUTA MIZUNO $^{1,2}$, KIMIAKI HATTORI ${ }^{1}$, KOHEI TANIGUCHI ${ }^{2,3}$, KEITARO TANAKA ${ }^{2}$, \\ KAZUHISA UCHIYAMA ${ }^{2}$ and YOSHINOBU HIROSE ${ }^{1}$
}

Departments of ${ }^{1}$ Pathology, ${ }^{2}$ General and Gastroenterological Surgery, and ${ }^{3}$ Translational Research Program, Osaka Medical College, Takatsuki, Osaka 569-8686, Japan

Received May 24, 2019; Accepted January 21, 2020

DOI: $10.3892 / 01.2020 .11390$

\begin{abstract}
The high expression of metabolic enzymes, including glutaminase (GA) and lactate dehydrogenase A (LDHA), which contribute to bioenergetics and biosynthesis of mammalian cells, has been identified in a variety of cancer types. The current study indicated intratumoral heterogeneity with respect to protein expression of the metabolic enzymes in colorectal cancer (CRC). GA protein expression was determined using immunohistochemistry in 98 cases of surgically resected T3 CRC. A total of 75 cases (74\%) exhibited moderate to strong immunopositivity of GA based on whole-section examination. A significant correlation was demonstrated between GA expression and clinicopathological features, including histological type and tumor budding in a patient population. Detailed histological analysis revealed the upregulation of GA protein expression at the invasive margin, including tumor budding of CRC tissues. Semi-quantitative examination revealed a significant difference in immunoexpression level of GA between the invasive margin and central CRC. However, LDHA expression exhibited an opposite pattern, with expression elevated at the center and significantly decreased at the tumors invasive margin. Immunohistochemical expression of another glycolytic enzyme hexokinase II was equivalent in both regions. Furthermore, gene silencing of GLSI, which encodes GA protein, and GA inhibitor treatment significantly inhibited cell growth of CRC cell lines. Therefore, the results of the present study demonstrated that the alteration in GA and LDHA expression is more prominent at the invasive margin, which involves tumor budding in CRC.
\end{abstract}

Correspondence to: Professor Yoshinobu Hirose, Department of Pathology, Osaka Medical College, 2-7 Daigaku-machi, Takatsuki, Osaka 569-8686, Japan

E-mail: hirose@osaka-med.ac.jp

Key words: colorectal cancer, budding, intratumoral heterogeneity, glutaminase, lactate dehydrogenase A, immunohistochemistry

\section{Introduction}

Currently, there is an increasing attention to altered energy metabolism in cancer. In normal cells, adequate oxygen usually inhibits glycolysis, allowing mitochondrial oxidative phosphorylation to generate ATP using glycolytic pyruvate (1). However, it is widely accepted that cancer cells increase, but do not decrease glycolysis even in an oxygen-sufficient environment. This notable phenomenon is well known as Warburg hypothesis (2), although the significance it confers to cancer cells has not been completely understood. In support of the hypothesis, multiple reports have shown that hexokinase II (HK2) and lactate dehydrogenase A (LDHA), which are critical enzymes, respectively catalyzing the initial and final steps of glycolysis, were overexpressed in many types of cancers (3-6). Recently, several lines of evidence have also revealed the new role of aerobic glycolysis, by which it does not only supply ATP but also yields biomass such as nucleotides, amino acids, or lipids necessary for cell proliferation (7).

Besides glucose, the mammalian cells can use other nutrients for energy production. Such alternatives include amino acids, especially glutamine, which is the most abundant amino acid in mammals (8). The oxidizing pathway of glutamine, a phenomenon known as glutaminolysis, begins with conversion of glutamine to glutamate by glutaminase (GA). Glutaminolysis shares several steps with TCA cycle, which leads to the recognition that glutamine is a source of energy generation (9). For cancer-specific metabolism, increased glutaminolysis is considered an important hallmark (10). Clinicopathological examination using immunohistochemistry showed that there was a relationship between increased GA expression and the malignant property of colorectal cancer (CRC) (11). There are two isoenzymes of GA, as denoted by the kidney and the liver-types encoded by $G L S 1$ and $G L S 2$ genes, respectively (12). In previous studies, the kidney-type of GA was indicated to play a role in facilitating tumorigenesis (13).

Tumor budding is defined as the presence of single malignant cells or small clusters composed of fewer than five cells at the invasive margin of tumors (14). Numerous evidence has shown that tumor budding is associated with adverse outcome, such as lymph node and distant metastasis of CRC (15). From the biological point of view, it is believed that this morphological feature is closely related to epithelial-mesenchymal transition (16). 
However, the metabolic characteristics particularly for tumor budding at the invasive margin remain unexplored.

In the present study, we investigated the immunohistochemical expression of energy-associated enzymes such as GA, LDHA, and HK2 in CRC, and we discussed the potential role of the metabolic alterations specifically at the invasive margin including tumor budding.

\section{Materials and methods}

Patients and tumor materials. Ninety-eight formalin-fixed and paraffin-embedded specimens of surgically resected T3 CRC, diagnosed at the Division of Pathology of Osaka Medical College hospital in 2013 were evaluated. Excluded were patients receiving chemotherapy or radiation therapy prior to surgery. Clinical data were obtained by reviewing patients' medical records. Pathological stages were determined according to American Joint Committee on Cancer 7th edition criteria for tumor staging (17). The primary sites of CRC were divided into right and left, with the splenic flexure as the dividing point. This study was approved by the Institutional Review Board (IRB) of Osaka Medical College (Approval no. 1571). The requirement for the written consent used for the research was waived by the IRB under the conditions being to use clinical data anonymously, to publicize the use of residual tissues, and to give participants the opportunity to opt out.

Histological and immunohistochemical analyses. Representative hematoxylin and eosin-stained sections were selected, and two pathologists reexamined all histopathological classification according to WHO criteria (18). Tumor budding was analyzed in accordance with the international evidence-based scoring system (19). Based on the bud count using 20x objective lens, those located principally at the invasive margin of tumors were categorized as follows: Low, $<5$ buds; intermediate, $5-9$ buds; high, $\geq 10$ buds. The invasive margin was defined as the five most distant cell layers from the central parts of tumors, and the tumor center was defined as the bulk of tumors excluding the invasive margin and the surface. Immunohistochemical staining was performed following the manufacturer's protocol (Vector Laboratories). Briefly, sections of $4 \mu \mathrm{m}$ thickness were cut from the representative paraffin block. After deparaffinization, endogenous peroxidase activity was quenched by $10 \mathrm{~min}$ incubation in $3 \%$ hydrogen peroxide solution. Then the sections were subjected to antigen retrieval using heat from pressure cooker, and were incubated with primary antibodies at room temperature for $30 \mathrm{~min}$. The primary antibodies used were as follows: GA, rabbit monoclonal antibody (Abcam), recognizing the kidney type and encoded by $G L S 1$, in a dilution of 1:400; LDHA, rabbit monoclonal antibody (Abcam) in a dilution of 1:2,000; HK2, rabbit monoclonal antibody (Proteintech) in a dilution of 1:100. Subsequently, all sections were kept in 3,3-diaminobenzidine as a chromogen for $5 \mathrm{~min}$. As negative controls, sections were treated by omitting the primary antibodies.

Immunohistochemical evaluation. We evaluated the immunostaining of GA protein initially on the entire tumor areas of the representative section. Immunoreactivity was classified using a modified method as previously described: Grade 1, immunopositive in $<25 \%$ of tumor cells; grade 2 , immunopositive in $25-50 \%$ of tumor cells; grade 3 , immunopositive in $>50 \%$ of tumor cells (20). In this setting, only the proportion of stained cells throughout the entire tumor areas was examined, and the intensity of staining was not considered. In addition, the superficial layers of the tumors were not examined because of vague immunopositivity, possibly due to erosive and necrotic changes. Grade 1 was regarded as negative and grades 2 and 3 as positive. For LDHA and HK2, immunohistochemical evaluation was performed similarly.

Semi-quantitative analysis of immunostaining. Considering intratumoral heterogeneity, we next conducted semi-quantitative analysis of immunoreactivity in the budding-positive cases ( $\geq 5$ buds). Briefly, we scanned up to 10 fields at the invasive margin to identify hotspots (with highest tumor budding) using x10 objective lens, and we examined immunohistochemical reactivity in the selected hotspot using x20 objective lens. For semi-quantification, a combination assessment of the proportion and intensity of immunostaining was performed $(20,21)$. In the selected field, we determined the proportion of zero, positive staining in $<25 \%$ of tumor cells; one, positive staining in $25-50 \%$ of tumor cells; two, positive staining in $51-75 \%$ of tumor cells; three, positive staining in more than $75 \%$ of tumor cells. Then we evaluated the intensity in the same field as follows: 0 , none; 1 , slight; 2 , abundant; 3 , strong. The product of the proportion and the intensity defined the score (0-9).

Cell lines and cell culture. DLD-1 and WiDr human CRC cell lines were obtained from Japanese Collection of Research Bioresources (JCRB) Cell Bank. Both cells were authenticated by short tandem repeated sequence profiling by JCRB, confirming that the WiDr was identical to HT-29 (22). Cells were cultured in RPMI-1640 (Invitrogen) with 10\% (v/v) heat-inactivated fetal bovine serum (Sigma-Aldrich). The temperature and atmosphere were $37^{\circ} \mathrm{C}$ and $95 \%$ air with $5 \%$ $\mathrm{CO}_{2}$, respectively.

Gene silencing assay. The siRNA for GLS1 (siR-GLS1, Silencer ${ }^{\circledR}$ Select Pre-Designed siRNA) was purchased from Life Technologies. The siRNA ID was s5840. The sequence of sense of siR-GLS1 was 5'-GAUUUGCUGUUCUAUACA Att-3' and that of antisense was 5'-UUGUAUAGAACAGCA AAUCtt-3'. Silencer Negative Control siRNA (Invitrogen) was used as the control for nonspecific effects. CRC cells were seeded in 6-well plates at a concentration of $0.5 \times 10^{5}$ cells per well on the day before transfection. The concentration of each siRNA was $10 \mathrm{nM}$. At $48 \mathrm{~h}$ after transfection, cell viability was determined through a dye exclusion test using trypan blue (Life Technologies).

GA Inhibition assay. BPTES (bis-2 (5-phenylacetamido-1,2,4-thiadiazol-2-yl) ethyl sulfide), an inhibitor of GA, was purchased from Selleck Chemicals. The concentrations of BPTES were chosen according to the information provided by Selleck and others $(23,24)$. To assess cell proliferation, CRC cells were seeded in 96-well plates at a concentration of $0.5 \times 10^{4}$ cells per well on the day before treatments. MTT(3-(4,5-dimethylthiazol-2-yl)-2,5-diphenyltetrazolium-bromide; Sigma-Aldrich) 
assay was performed according to the protocol described previously (25). In the assay, absorbance at $540 \mathrm{~nm}$ was measured using SH-1000Lab microplate reader (Corona Electric).

Western blot analysis. At $48 \mathrm{~h}$ after transfection, protein samples were extracted from the cells and western blot analysis was performed following the protocols described previously $(25,26)$. The materials used for the analysis were as follows: RIPA buffer (Thermo Fisher Scientific, Inc.), Protease Inhibitor Cocktail (Sigma-Aldrich), DC Protein assay kit (Bio-Rad), polyacrylamide gels (Wako Pure Chemical), PVDF membrane (Bio-Rad), and PVDF Blocking Reagent for Can Get Signal ${ }^{\circledR}$ (TOYOBO). The primary antibodies used were anti-GA (Abcam; EP7212), anti-LDHA (Abcam; EP1566Y), and anti- $\beta$-actin (Sigma-Aldrich; A2228). The secondary antibodies used were HRP-conjugated goat anti-rabbit and horse anti-mouse IgG (Cell Signaling Technology). The immunoblots were detected and visualized by Fusion-FX7 (Vilber Lourmat) with LuminataTM Forte Western HRP Substrate (Millipore). $\beta$-actin was used as an internal control.

cBioPortal data analysis. Data regarding genomic alterations of $G L S 1$ in patients with CRC were obtained through the cBioPortal for Cancer Genomics website (http://www.cbioportal. org/), which we accessed on October 27, 2019. The database of The Cancer Genome Atlas (TCGA) PanCancer atlas studies was used for cross-cancer analysis of mutations and copy number alterations (CNAs) of GLS1 (27-29). In addition, to enrich the number of CRC samples, six other studies of CRC (DFCI, Genentech, MSK 2014, CaseCCC, CPTAC-2, and MSK 2018) were chosen (30-35). All searches were performed in accordance with the cBioPortal online instructions.

Statistical analysis. The association between immunoexpression status of GA and clinicopathological characteristics was analyzed using chi-square test. Semi-quantitative scores of GA, LDHA, and HK2 were compared with Wilcoxon rank sum test. Kaplan-Meier curves were constructed to plot the probability of patient's overall survival, and the differences between survival distributions were analyzed with the log-rank test. For in vitro experiments that were performed in triplicates in each assay, the two-sided Student's t-test was used to determine the statistical significance of the differences. $\mathrm{P}<0.05$ was considered to indicate a statistically significant difference.

\section{Results}

Clinicopathological data. The clinicopathological background of all 98 cases of CRC is shown in Table I. The patients had a median age of 70.0 years and there were 56 males (57\%) and 42 females (43\%). There were 54 cases $(55 \%)$ in stage II, 39 cases $(40 \%)$ in stage III, and 5 cases $(5 \%)$ in stage IV. Histologic types were composed of 90 cases of usual adenocarcinoma (92\%) and 8 cases of variants including mucinous and micropapillary (8\%). The primary sites consisted of 31 cases (32\%) of right colon and 67 cases $(68 \%)$ of left colon. Fifty-five cases (56\%) were positive for lymph node and/or distant metastasis. For tumor budding, 65 cases $(66 \%)$ were categorized as low and 33 cases (34\%) as intermediate to high tumor budding.
Immunoexpression of $G A$ in the entire areas of $C R C$. First, we assessed immunohistochemical expression of GA based on whole-section examination. Seventy-five (74\%) of 98 cases were positive for immunostaining of GA (grades 2 and 3 ). As described in Table I, the status of GA expression showed statistically significant association with histological type $(\mathrm{P}=0.0264)$ and tumor budding $(\mathrm{P}=0.0448)$, but not with metastasis $(\mathrm{P}=0.3260)$ in our patient population. In addition, we scrutinized the relationship between GA status and patient prognosis. Kaplan-Meier analysis (Fig. 1) revealed that there was no significant association between GA expression and patient prognosis $(\mathrm{P}=0.93)$. Next, we focused on the distribution of GA immunopositivity in cancer tissues because of the positive correlation between GA expression and tumor budding (Fig. 2A). As shown in Fig. 2B and C, there was a tendency towards enhanced immunoreactivity of GA protein at the invasive margin along with tumor budding. This increased expression was more conspicuous in low GA expression cases (grade 1) with intermediate to high tumor budding (3 cases). Such cases exhibited increased immunopositivity of GA more robustly and preferentially at the invasive margin, containing tumor budding, despite its partial and arbitrary expression in the center (Fig. 2D and E).

Semi-quantitative analysis of GA expression. To elucidate the correlation between GA expression and tumor budding, we extracted and focused on the subgroup of intermediate to high-budding cases $(n=33)$. In this subgroup, immunoreactivity of GA was semi-quantitatively assessed by comparing the invasive margin including tumor budding with the center region (6). Consequently, the semi-quantitative scores of GA immunoexpression were $5.8 \pm 3.0$ for the invasive margin and $3.7 \pm 2.6$ for the center (Table II). Statistically, the immunoexpression of GA at the margin was significantly higher than that of the center $(\mathrm{P}=0.0005)$.

Immunoexpression of LDHA and HK2. In the subgroup of intermediate/high budding cases, we performed additional immunohistochemistry of LDHA and HK2. In 26 of 33 cases (79\%), we confirmed positive immunoreactivity for LDHA in the entire tumor areas on the sections. Notably, our immunohistochemistry indicated that the immunoexpression of LDHA at the invasive margin with tumor budding was likely to be weak compared to that in the center (Fig. 3A and B). Semi-quantitative analysis revealed that LDHA scores were $0.4 \pm 0.6$ for the invasive margin and $2.5 \pm 2.1$ for the center (Table II). Statistically, LDHA score of the invasive margin was significantly lower than that of the center $(\mathrm{P}<0.001)$. Further, we frequently observed opposite trends for LDHA and GA expressions at the periphery of identical cancer nests, with weaker staining for LDHA and increased staining for GA (Fig. 3C-F). Next, we evaluated the expression and distribution of HK2 in the same way. Our immunohistochemistry showed positive immunoreactivity of HK2 in 32 of 33 cases (97\%). However, there was no significant difference in HK2 expression between the margin and the center (Table II).

Genomic alterations of GLSI in a range of cancer types and CRC databases. To elucidate whether the heterogeneous expressions of the proteins were due to gene mutation or amplification, we first examined the genomic alterations of GLSI in the 
Table I. Association between clinicopathological features and GA immunohistochemical status in all cases $(n=98)$.

\begin{tabular}{|c|c|c|c|c|c|}
\hline \multirow[b]{2}{*}{ Variable } & \multirow[b]{2}{*}{ No. of cases $(\%)$} & \multicolumn{3}{|c|}{ GA expression } & \multirow[b]{2}{*}{ P-value } \\
\hline & & Grade 1 & Grade 2 & Grade 3 & \\
\hline Sex & & & & & 0.2529 \\
\hline Male & $56(57)$ & 15 & 14 & 27 & \\
\hline Female & $42(43)$ & 8 & 17 & 17 & \\
\hline Age & & & & & 0.7472 \\
\hline$<59$ & $18(18)$ & 3 & 6 & 9 & \\
\hline$\geq 60$ & $80(82)$ & 20 & 25 & 35 & \\
\hline BMI & & & & & 0.1574 \\
\hline$\geq 25.0$ & $14(14)$ & 5 & 6 & 3 & \\
\hline$\leq 25.0$ & $84(86)$ & 18 & 25 & 41 & \\
\hline TNM stage & & & & & 0.4940 \\
\hline II & $54(55)$ & 15 & 14 & 25 & \\
\hline III & $39(40)$ & 8 & 15 & 16 & \\
\hline IV & $5(5)$ & 0 & 2 & 3 & \\
\hline Histological type & & & & & 0.0264 \\
\hline Usual & $90(92)$ & 20 & 26 & 44 & \\
\hline Variants & $8(8)$ & 3 & 5 & 0 & \\
\hline Primary site & & & & & 0.5813 \\
\hline Right & $31(32)$ & 9 & 8 & 14 & \\
\hline Left & $67(68)$ & 14 & 23 & 30 & \\
\hline Lymphatic invasion & & & & & 0.7044 \\
\hline Absent & $9(9)$ & 1 & 2 & 6 & \\
\hline Present (low) & $67(68)$ & 17 & 21 & 29 & \\
\hline Present (high) & $22(23)$ & 5 & 8 & 9 & \\
\hline Vascular invasion & & & & & 0.0903 \\
\hline Absent & $16(16)$ & 6 & 7 & 3 & \\
\hline Present (low) & $65(66)$ & 16 & 18 & 31 & \\
\hline Present (high) & $17(18)$ & 1 & 6 & 10 & \\
\hline Metastasis & & & & & 0.3260 \\
\hline Negative & $54(55)$ & 15 & 14 & 25 & \\
\hline Positive & $44(45)$ & 8 & 17 & 19 & \\
\hline Tumor budding & & & & & 0.0448 \\
\hline Low & $65(66)$ & 20 & 20 & 25 & \\
\hline Intermediate/High & $33(34)$ & 3 & 11 & 19 & \\
\hline
\end{tabular}

GA, glutaminase; BMI, body mass index.

TCGA PanCancer Atlas Studies (10967 cases in 32 studies) for cross-cancer analysis. As shown in Fig. 4A, the overall rate of the genomic alterations was low in various cancer types, and the rates of mutations and CNAs of GLS1 were $0.84 \%$ ( 5 cases) and $0 \%$, respectively, in $594 \mathrm{CRC}$ cases. Next, we investigated the GLS1 alterations in 6 other CRC studies to enrich the number of samples. As shown in Fig. 4B, the ratio of GLS1 mutations was $0.93 \%$ (9 cases) in 964 CRC cases. As for CNAs of GLS1, no positive case was found in the CRC databases (1239 cases).

Suppression of GLS1 inhibited CRC cells growth. Finally, we further confirmed whether GLS1 affected CRC cell growth. Gene silencing effects on GLS1 by siRNA-GLS1 were examined in two CRC cell lines, DLD-1 and WiDr cells (Figs. 5A and S1). As shown in Fig. 5B, knockdown of GLS1 significantly inhibited CRC cells growth. BPTES, which was known as a GA inhibitor, also suppressed cell growth of both CRC cell lines in a dose-dependent manner (Fig. 5C).

\section{Discussion}

Although the relationship between cancer and altered metabolism is not new, the interest in cancer metabolism has been rekindled, partly because of the prevalence of metabolic-based 


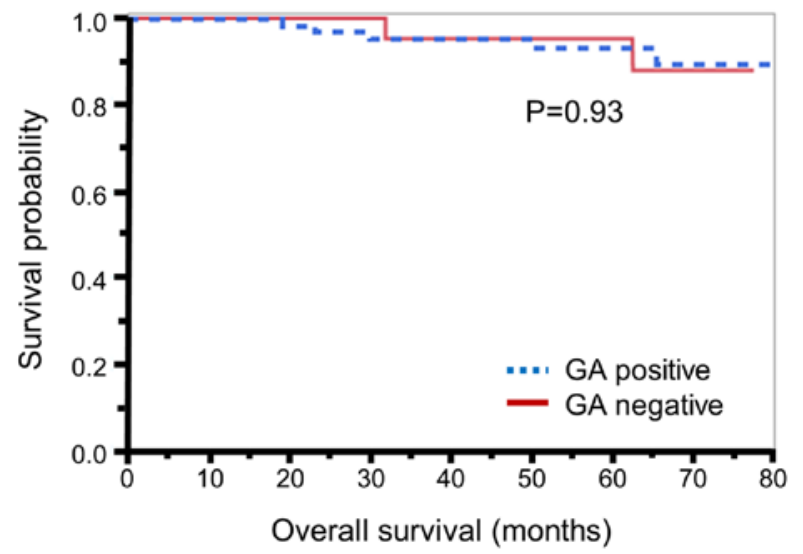

Figure 1. Kaplan-Meier overall survival curve in 98 patients with pT3 colorectal adenocarcinoma. There was no significant association between GA expression and patients prognosis $(\mathrm{P}=0.93)$. GA, glutaminase.
A

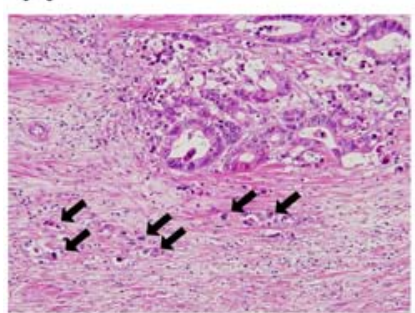

C

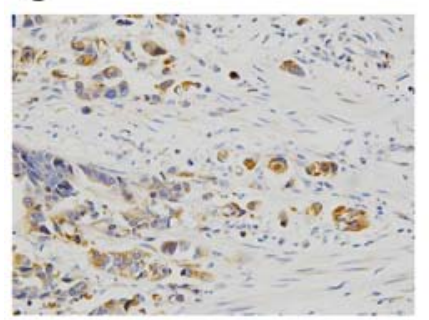

E

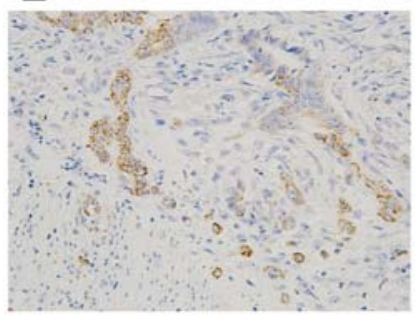

B

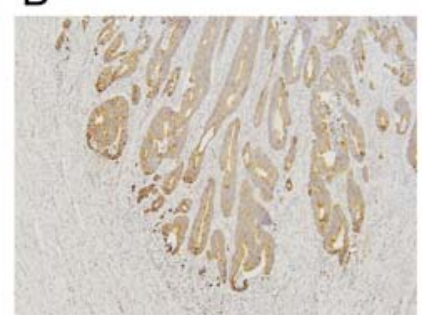

D

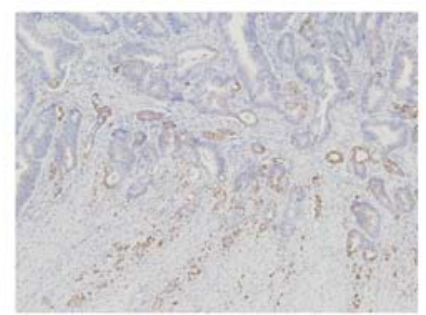

A

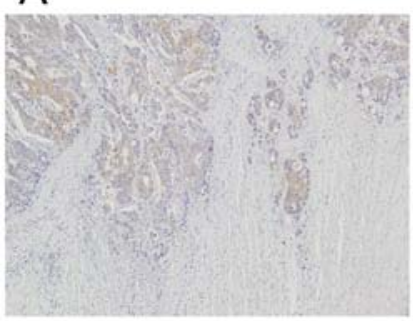

C

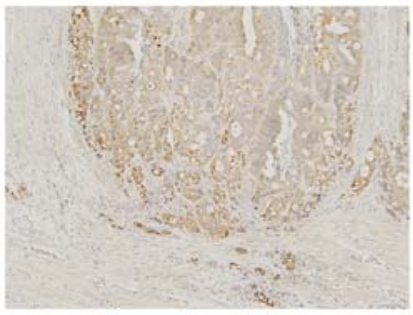

$\mathrm{E}$

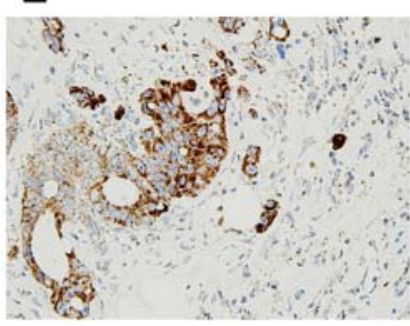

B

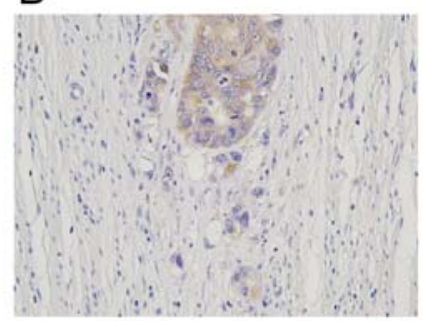

D

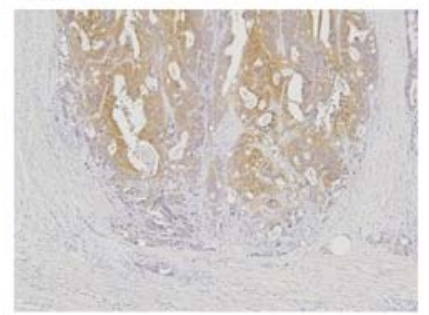

F

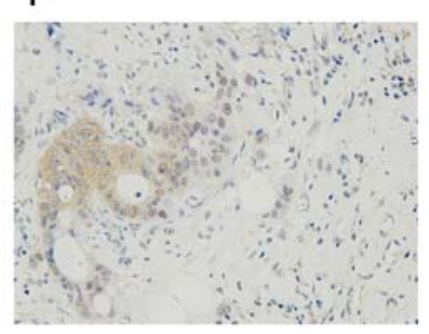

Figure 3. Immunohistochemistry of LDHA. (A) Immunohistochemistry of LDHA (original magnification, x100), and (B) the invasive margin, including tumor budding, indicating minimal expression (original magnification, x400). (C) Increased GA expression and (D) weaker LDHA expression at the periphery of the same cancer nest (original magnification, x100). Representative images of the protein expressions of (E) GA and (F) LDHA using serial sections (original magnification, $\mathrm{x} 400$ ). LDHA, lactate dehydrogenase A; GA, glutaminase. tumor budding. (haematoxylin and eosin; original magnification, x200) (B) Immunohistochemistry of GA (original magnification, $\mathrm{x} 100$ ), and (C) tumor budding at the invasive margin with prominent expression of GA protein (original magnification, $x 400$ ). (D) An example of GA-negative CRC preserving high levels of GA expression at the invasive margin. The upper and lower parts of the image indicate the center and invasive front, respectively (original magnification, x100). (E) Strong expression of GA at the invasive margin with tumor buds in GA-negative case (original magnification, $x 400$ ). CRC, colorectal cancer; GA, glutaminase.

imaging modalities, such as positron emission tomography, for detecting cancer (1). Much attention has been attracted to the relationship by the identification and characterization of driver mutations in metabolic enzymes such as isocitrate
Table II. Semiquantitative result of immunohistochemical expression of GA, LDHA and HK2 in the margin and center of CRC.

\begin{tabular}{lccc}
\hline Score & Margin & Center & P-value \\
\hline GA & $5.8 \pm 3.0$ & $3.7 \pm 2.6$ & 0.0005 \\
LDHA & $0.4 \pm 0.6$ & $2.5 \pm 2.1$ & $<0.001$ \\
HK2 & $7.5 \pm 2.8$ & $7.8 \pm 2.7$ & 0.0832
\end{tabular}

Values are mean \pm standard deviation; GA, glutaminase; LDHA, lactate dehydrogenase $\mathrm{A}$; $\mathrm{HK} 2$, hexokinase II; CRC, colorectal cancer. dehydrogenase in several cancers (36). To date, it is more important to consider the metabolic trait of cancer from basic research to clinical application, especially in the fields of genome-based tumor diagnosis or molecular-targeted therapy. However, tumor budding has been established as a surrogate marker for malignant grade and prognosis in clinical practice $(14,15)$. The explorations involving tumor budding with epithelial-mesenchymal transition and subsequent causation of metastasis have grown and attracted more attention (37), 
A

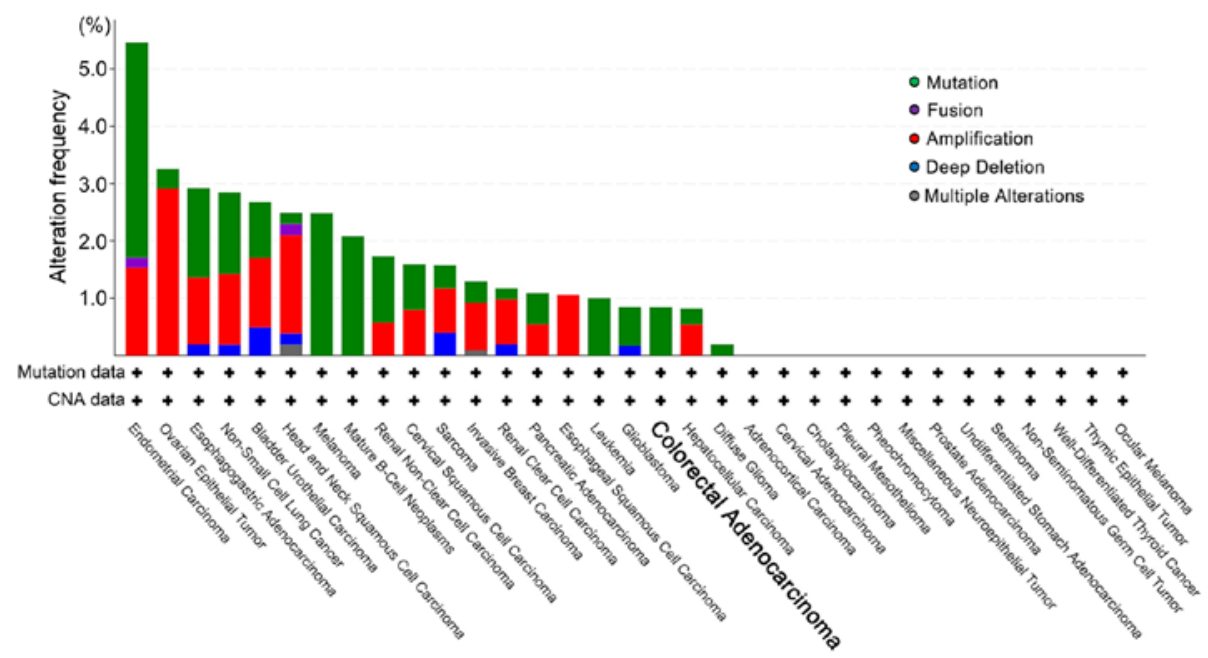

B

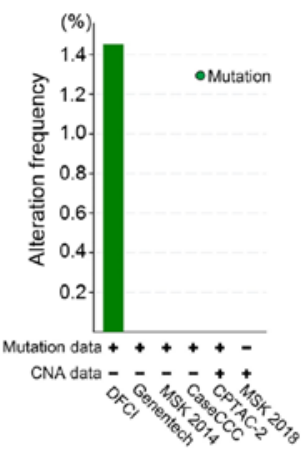

Figure 4. Genomic alterations of GLS1 in the data sets at the cBioPortal for Cancer Genomics. (A) Cross-cancer analysis of GLS1 using the dataset of TCGA Pan-Cancer Atlas Studies. Green, mutation; purple, fusion; red, amplification; blue, deep deletion; and gray, multiple alteration. (B) Genomic alterations of GLS1 in large datasets of CRC. TCGA, The Cancer Genome Atlas; CNA, copy number alterations.

A

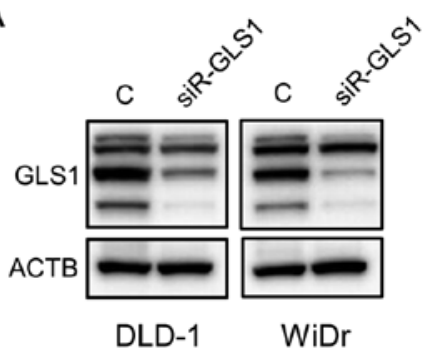

C

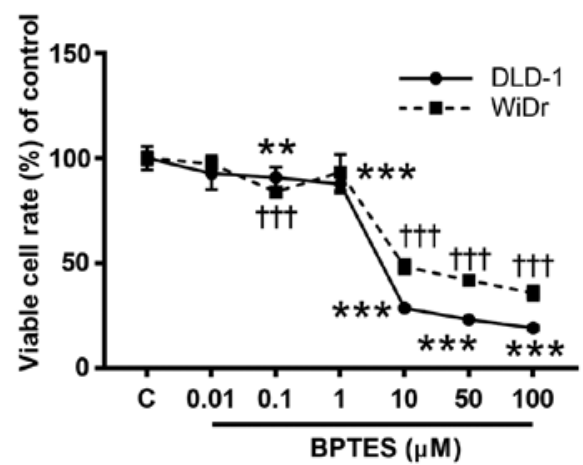

B
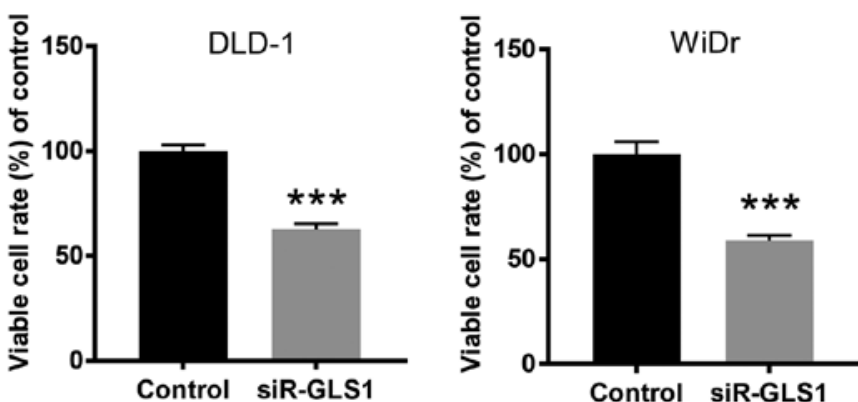

Figure 5. The effect of suppressive GLS1 gene on CRC cells. (A) The protein expression levels of GLS1 in siR-GLS1-treated CRC cells. (B) Cell viability after transfection with siR-GLS1 in DLD-1 and WiDr cells. The concentration of siR-GLS1 was $10 \mathrm{nM}$ and the effects were assessed at $48 \mathrm{~h}$ after transfection. (C) Cell viability after treatment with GA inhibitor (BPTES) in DLD-1 and WiDr cells. The effects were assessed at $48 \mathrm{~h}$ after treatments. Results are expressed as percentages of cell number in the untreated control (mean \pm standard deviation); ${ }^{* *} \mathrm{P}<0.01 ;{ }^{* * * *} \mathrm{P}<0.001 ;{ }^{\dagger \dagger \dagger} \mathrm{P}<0.001$. CRC, colorectal cancer; si, small interfering; C, control; GA, glutaminase.

whereas those of metabolic features remain limited. Therefore, the objective of our current study is to elucidate the tumor budding-specific metabolism.

On the basis of the significant association between GA protein expression and tumor budding status, we demonstrated for the first time that GA expression is augmented more preferentially at the invasive margin, including the tumor buds, in CRC. When intratumoral heterogeneity was excluded, this observation is generally in line with those in previous studies, which concluded that the GA expression was upregulated in CRC (11). As we found mutations a small percentage and no CNAs of GLS1 in CRC in the cBioPortal data analysis, our 
commonly recognized finding could not be due to such genomic changes in GA. Moreover, this is of great interest because of its functional implication at the invasive margin, containing tumor budding of CRC. At first, it is reasonable to suggest that upregulation of GA is likely to be due to the requirement of glutamine. This may indicate that the marginal region appears more glutamine-addicted in CRC tissues (38). In addition, this suggests a possible occurrence of glutamine anaplerosis at the invasive margin, involving tumor budding (8). Anaplerosis is the replenishing process to have a matching influx of TCA cycle intermediates, which are consumed and lacking (39). Glutamine is regarded as a fundamental source (40) and therefore, our data may imply that the marginal cells along with tumor budding need more biomass via glutamine anaplerosis to proliferate, invade, and metastasize. Interestingly, our in vitro assay showed that forced suppression of GLS1 gene expression clearly inhibited the growth of CRC cells. This observation from our assays, though still small and insufficient, warrants further investigation in terms of possible GA-targeted therapy for CRC patients.

In contrast, LDHA expression exhibited the opposite pattern in the majority of cases, with its expression increased at the center and significantly, but unexpectedly, weaker at the invasive margin of the tumors. LDH is a tetrameric enzyme responsible for glycolysis and they are of two types: Muscle/anaerobic (LDHA) and heart/aerobic (LDHB) (41). It is widely appreciated that aberrantly high expression of LDHA, but not LDHB, is crucial for Warburg effect and carcinogenesis (3-5). In this perspective, unless considering intratumoral heterogeneity, our present data are consistent with previous reports (3). As far as intratumoral heterogeneity is concerned, this is the first demonstration in terms of spatially heterogeneous expression of LDHA in CRC tissues, to the best of our knowledge. At the same time, this finding raises a question of why reduced LDHA expression occurs at the invasive margin involving tumor budding. The reason remains unknown, but it may be simply explained by the microenvironment of relative non-hypoxic. Since hypoxia can lead to upregulation of LDHA (42), its downregulation may be reflected in non-hypoxic condition of the invasive margin in comparison with the center. Another explanation can be provided by a recent report showing that a certain condition such as arginine deprivation upregulated glutamine anaplerosis, as well as inhibited Warburg effect, in argininosuccinate synthetase 1-deficient cancers (43). To begin with, LDHA regulation remains complex and far from being completely understood, since numerous genes have been reported to be related to LDHA expression and activity (44). Thus, our present finding warrants further investigation to reveal the role and mechanism of LDHA expression at the marginal area of CRC.

In recent years, there has been increased awareness and scrutiny of intratumoral heterogeneity, in view of morphological, genetic, and biological characteristics (45-47). To detect, analyze, and interpret this, we should keep in mind that comprehensive and comparative approaches toward cancer tissues are required. For this purpose, there are several issues concerned particularly about the methodology. First, high-throughput screenings such as tissue microarray, which is familiar at present, appear not suitable as a method for evaluating heterogeneity of tumors histopathologically, because they assess small and partial regions of tumor tissues sampled $(48,49)$. Second, the evaluation of immunohistochemistry in many published reports has been done using the so-called 'positive or negative' method, in which stainability was determined in a tumor as a whole. In such dichotomy, there should be a tendency to underestimate heterogeneity of tumors, since the assessment occasionally misses or omits minor portions $(48,50,51)$.

The present study has several limitations. The number of the samples was small because we selected only pT3 CRC cases. Further study that includes different categories and various histopathological subtypes is required. In addition, the in vitro experiments in the present study were preliminary. To account for the association between GA and LDHA expression at the invasive margin of CRC, further investigation such as in vitro experiments, animal model studies, or metabolic analysis should be conducted.

In conclusion, we described herein a novel immunohistological-based alteration in GA and LDHA expression at the invasive margin together with tumor budding, based on significant correlation between GA expression and tumor budding. These results are the first step towards extending the investigation into the mechanisms underlying this intriguing alteration and its effect on metabolic enzymes.

\section{Acknowledgements}

The authors would like to thank Ms Manami Shimoguchi (Department of Pathology, Osaka Medical College) for assistance in performing immunohistochemistry and data analysis.

\section{Funding}

The present study was partially supported by JSPS KAKENHI (grant nos. JP25430136 and JP16H07344).

\section{Availability of data and materials}

The datasets used and/or analyzed during the present study are available from the corresponding author on reasonable request.

\section{Authors' contributions}

$\mathrm{YH}$ and $\mathrm{KU}$ designed the experiment. KoT and KeT collected clinicopathological information from patients. YM and $\mathrm{KH}$ conducted immunohistochemistry. KH and YH performed evaluation of the immunohistochemistry slides and data analysis. KoT performed in vitro experiments and cBioPortal data analysis. YM, KoT and YH wrote the manuscript. All authors have read and approved the final manuscript.

\section{Ethics approval and consent to participate}

The present study was approved by the Institutional Review Board of Osaka Medical College. The requirement for written consent was waived.

\section{Patient consent for publication}

Not applicable. 


\section{Competing interests}

The authors declare that they have no competing interests.

\section{References}

1. Gatenby RA and Gillies RJ: Why do cancers have high aerobic glycolysis? Nat Rev Cancer 4: 891-899, 2004.

2. Warburg O: On the origin of cancer cells. Science 123: 309-314, 1956

3. Koukourakis MI, Giatromanolaki A, Sivridis E, Gatter KC and Harris AL; Tumour Angiogenesis Research Group: Lactate dehydrogenase 5 expression in operable colorectal cancer: Strong association with survival and activated vascular endothelial growth factor pathway-a report of the Tumour Angiogenesis Research Group. J Clin Oncol 24: 4301-4308, 2006.

4. Rong Y, Wu W, Ni X, Kuang T, Jin D, Wang D and Lou W: Lactate dehydrogenase $\mathrm{A}$ is overexpressed in pancreatic cancer and promotes the growth of pancreatic cancer cells. Tumor Biol 34: 1523-1530, 2013.

5. Yao F, Zhao T, Zhong C, Zhu J and Zhao H: LDHA is necessary for the tumorigenicity of esophageal squamous cell carcinoma. Tumor Biol 34: 25-31, 2013

6. Hamabe A, Yamamoto H, Konno M, Uemura M, Nishimura J, Hata T, Takemasa I, Mizushima T, Nishida N, Kawamoto K, et al: Combined evaluation of hexokinase 2 and phosphorylated pyruvate dehydrogenase-E1 $\alpha$ in invasive front lesions of colorectal tumors predicts cancer metabolism and patient prognosis. Cancer Sci 105: 1100-1108, 2014

7. Vander Heiden MG, Cantley LC and Thompson CB Understanding the Warburg effect: The metabolic requirements of cell proliferation. Science 324: 1029-1033, 2009.

8. Daye D and Wellen KE: Metabolic reprogramming in cancer Unraveling the role of glutamine in tumorigenesis. Semin Cell Dev Biol 23: 362-369, 2012.

9. Márquez J, de la Oliva AR, Matés JM, Segura JA and Alonso FJ: Glutaminase: A multifaceted protein not only involved in generating glutamate. Neurochem Int 48: 465-471, 2006.

10. Yang L, Venneti S and Nagrath D: Glutaminolysis: A hallmark of cancer metabolism. Annu Rev Biomed Eng 19: 163-194, 2017.

11. Huang F, Zhang Q, Ma H, Lv Q and Zhang T: Expression of glutaminase is upregulated in colorectal cancer and of clinical significance. Int J Clin Exp Pathol 7: 1093-1100, 2014.

12. Curthoys NP and Watford M: Regulation of glutaminase activity and glutamine metabolism. Annu Rev Nutr 15: 133-159, 1995.

13. Van Den Heuvel APJ, Jing J, Wooster RF and Bachman KE: Analysis of glutamine dependency in non-small cell lung cancer: GLS1 splice variant GAC is essential for cancer cell growth Cancer Biol Ther 13: 1185-1194, 2012.

14. Ueno H, Murphy J, Jass JR, Mochizuki H and Talbot IC: Tumour budding as an index to estimate the potential of aggressiveness in rectal cancer. Histopathol 40: 127-132, 2002.

15. Prall F: Tumour budding in colorectal carcinoma. Histopathol 50 $151-162,2007$

16. Yang $\mathbf{J}$ and Weinberg RA: Epithelial-mesenchymal transition: At the crossroads of development and tumor metastasis. Dev Cell 14: 818-829, 2008.

17. Edge SB and Compton CC: The American joint committee on cancer: The 7th edition of the AJCC cancer staging manual and the future of TNM. Ann Surg Oncol 17: 1471-1474. 2010

18. Hamilton SR, Bosman FT, Boffetta P, Ilyas M, Morreau H, Nakamura SI, Quirke P, Riboli E and Sobin LH: Carcinoma of the colon and rectum. In: WHO classification of tumours of the digestive system. Bozman FT, Carneiro F, Hruban RH and Theise ND (eds.) IARC Press, Lyon, pp134-146, 2010.

19. Lugli A, Kirsch R, Ajioka Y, Bosman F, Cathomas G, Dawson H, El Zimaity H, Fléjou JF, Hansen TP, Hartmann A, et al: Recommendations for reporting tumor budding in colorectal cancer based on the International Tumor Budding Consensus Conference (ITBCC) 2016. Modern Pathol 30: 1299-1311, 2017.

20. GirgisH,MasuiO, WhiteNM,Scorilas A, RotondoF, Seivwright A Gabril M, Filter ER, Girgis AH, Bjarnason GA, et al: Lactate dehydrogenase $\mathrm{A}$ is a potential prognostic marker in clear cell renal cell carcinoma. Mol Cancer 13: 101, 2014.

21. Ondruschka C,Buhtz P, Motsch C,Freigang B, Schneider-Stock R Roessner A and Boltze C: Prognostic value of MMP-2, -9 and TIMP-1,-2 immunoreactive protein at the invasive front in advanced head and neck squamous cell carcinomas. Pathol Res Pract 198: 509-515, 2002 .
22. Chen TR, Drabkowski D, Hay RJ, Macy M and Peterson W Jr: WiDr is a derivative of another colon adenocarcinoma cell line, HT-29. Cancer Genet Cytogenet 27: 125-134, 1987.

23. SeltzerMJ,BennettBD, Joshi AD, Gao P, Thomas AG,Ferraris DV, Tsukamoto T, Rojas CJ, Slusher BS, Rabinowitz JD, et al: Inhibition of glutaminase preferentially slows growth of glioma cells with mutant IDH1. Cancer Res 70: 8981-8987, 2010.

24. Xiang Y, Stine ZE, Xia J, Lu Y, O'Connor RS, Altman BJ, Hsieh AL, Gouw AM, Thomas AG, Gao P, et al: Targeted inhibition of tumor-specific glutaminase diminishes cell-autonomous tumorigenesis. J Clin Invest 125: 2293-2306, 2015.

25. Kawaguchi N, Tashiro K, Taniguchi K, Kawai M, Tanaka K, Okuda J,Hayashi M and Uchiyama K: Nogo-B (Reticulon-4B) functions as a negative regulator of the apoptotic pathway through the interaction with c-FLIP in colorectal cancer cells. Biochim Biophys Acta Mol Basis Dis 1864: 2600-2609, 2018.

26. Kagota S, Taniguchi K, Lee SW, Ito Y, Kuranaga Y, Hashiguchi Y, Inomata Y, Imai Y, Tanaka R, Tashiro K, et al: Analysis of extracellular vesicles in gastric juice from gastric cancer patients. Int J Mol Sci 20: pii: E953, 2019.

27. Cerami E, Gao J, Dogrusoz U, Gross BE, Sumer SO, Aksoy BA, Jacobsen A, Byrne CJ, Heuer ML, Larsson E, et al: The cBio cancer genomics portal: An open platform for exploring multidimensional cancer genomics data. Cancer Discov 2: 401-404, 2012.

28. Gao J, Aksoy BA, Dogrusoz U, Dresdner G, Gross B, Sumer SO, Sun Y, Jacobsen A, Sinha R, Larsson E, et al: Integrative analysis of complex cancer genomics and clinical profiles using the cBioPortal. Sci Signal 6: pl1, 2013.

29. Cancer Genome Atlas Network: Comprehensive molecula characterization of human colon and rectal cancer. Nature 487: 330-337, 2012.

30. Giannakis M, Mu XJ, Shukla SA, Qian ZR, Cohen O, Nishihara R, Bahl S, Cao Y, Amin-Mansour A, Yamauchi M, et al: Genomic correlates of immune-cell infiltrates in colorectal carcinoma. Cell Rep 15: 857-865, 2016

31. Seshagiri S, Stawiski EW, Durinck S, Modrusan Z, Storm EE, Conboy CB, Chaudhuri S, Guan Y, Janakiraman V, Jaiswal BS, et al: Recurrent R-spondin fusions in colon cancer. Nature 488: 660-664, 2012.

32. Brannon AR, Vakiani E, Sylvester BE, Scott SN, McDermott G, Shah RH, Kania K, Viale A, Oschwald DM, Vacic V, et al: Comparative sequencing analysis reveals high genomic concordance between matched primary and metastatic colorectal cancer lesions. Genome Biol 15: 454, 2014.

33. Yaeger R, Chatila WK, Lipsyc MD, Hechtman JF, Cercek A, Sanchez-Vega F, Jayakumaran G, Middha S, Zehir A Donoghue MTA, et al: Clinical sequencing defines the genomic landscape of metastatic colorectal cancer. Cancer Cell 33: 125-133.e3, 2018.

34. Guda K, Veigl ML, Varadan V, Nosrati A, Ravi L, Lutterbaugh J, Beard L, Willson JK, Sedwick WD, Wang ZJ, et al: Novel recurrently mutated genes in African American colon cancers. Proc Natl Acad Sci USA 112: 1149-1154, 2015

35. Vasaikar S, Huang C, Wang X, Petyuk VA, Savage SR, Wen B, Dou Y,Zhang Y, Shi Z, Arshad OA, et al: Proteogenomic analysis of human colon cancer reveals new therapeutic opportunities. Cell 177: 1035-1049.e19, 2019.

36. Cairns RA, Harris IS and Mak TW: Regulation of cancer cell metabolism. Nature Rev Cancer 11: 85-95, 2011.

37. Brabletz T, Hlubek F, Spaderna S, Schmalhofer O, Hiendlmeyer E, Jung A and Kirchner T: Invasion and metastasis in colorectal cancer: Epithelial-mesenchymal transition, mesenchymal-epithelial transition, stem cells and beta-catenin. Cells Tissues Organs 179: 56-65, 2005.

38. Reitzer LJ, Wice BM and Kennell D: Evidence that glutamine, not sugar, is the major energy source for cultured HeLa cells. J Biol Chem 254: 2669-2676, 1979.

39. Owen OE, Kalhan SC and Hanson RW: The key role of anaplerosis and cataplerosis for citric acid cycle function. J Biol Chem 277: 30409-30412, 2002.

40. DeBerardinis RJ, Lum JJ, Hatzivassiliou G and Thompson CB The biology of cancer: Metabolic reprogramming fuels cell growth and proliferation. Cell Metab 7: 11-20, 2008.

41. Duka T, Collins Z, Anderson SM, Raghanti MA, Ely JJ, Hof PR, Wildman DE, Goodman M, Grossman LI and Sherwood CC: Divergent lactate dehydrogenase isoenzyme profile in cellular compartments of primate forebrain structures. Mol Cell Neurosci 82: 137-142, 2017. 
42. Langhammer S, Najjar M, Hess-Stumpp H and Thierauch KH LDH-A influences hypoxia-inducible factor $1 \alpha(\mathrm{HIF}-1 \alpha)$ and is critical for growth of HT29 colon carcinoma cells in vivo. Targ Oncol 6: 155-162, 2011.

43. Kremer JC, Prudner BC, Lange SES, Bean GR, Schultze MB Brashears CB, Radyk MD, Redlich N, Tzeng SC, Kami K, et al: Arginine deprivation inhibits the Warburg effect and Upregulates glutamine anaplerosis and serine biosynthesis in ASS1-deficient cancers. Cell Rep 18: 991-1004, 2017.

44. Valvona CJ, Fillmore HL, Nunn PB and Pilkington GJ: The regulation and function of lactate dehydrogenase $\mathrm{A}$ : Therapeutic potential in brain tumor. Brain Pathol 26: 3-17, 2016.

45. Losi L, Baisse B, Bouzourene $\mathrm{H}$ and Benhattar J: Evolution of intratumoral genetic heterogeneity during colorectal cancer progression. Carcinogenesis 26: 916-922, 2005.

46. Brattain MG, Fine WD, Khaled FM, Thompson $\mathrm{J}$ and Brattain DE: Heterogeneity of malignant cells from a human colonic carcinoma. Cancer Res 41: 1751-1756, 1981.

47. Hu J, Locasale JW, Bielas JH, O'sullivan J, Sheahan K, Cantley LC, Vander Heiden MG and Vitkup D: Heterogeneity of tumor-induced gene expression changes in the human metabolic network. Nat Biotechnol 31: 522-529, 2013.
48. Nassar A, Radhakrishnan A, Cabrero IA, Cotsonis GA and Cohen C: Intratumoral heterogeneity of immunohistochemical marker expression in breast carcinoma: A tissue microarray-based study. Appl Immunohistochem Mol Morphol 18: 433-441, 2010.

49. Böger C, Behrens HM and Röcken C: Ki67-An unsuitable marker of gastric cancer prognosis unmasks intratumoral heterogeneity. J Surg Oncol 113: 46-54, 2016.

50. Allott EH, Geradts J, Sun X, Cohen SM, Zirpoli GR, Khoury T, Bshara W, Chen M, Sherman ME, Palmer JR, et al: Intratumoral heterogeneity as a source of discordance in breast cancer biomarker classification. Breast Cancer Res 18: 68, 2016.

51. Jakobsen JN, Santoni-Rugiu E, Ravn J and Sørensen JB: Intratumour variation of biomarker expression by immunohistochemistry in resectable non-small cell lung cancer. Eur J Cancer 49: 2494-2503, 2013.

This work is licensed under a Creative Commons Attribution-NonCommercial-NoDerivatives 4.0 International (CC BY-NC-ND 4.0) License. 